Supporting Information

\title{
Insight into the Determining Effect of Carbon Support Properties on Anchoring Active Sites in Fe-N-C Catalysts toward the Oxygen Reduction Reaction
}

Jing $\mathrm{He}^{\dagger}$, Tianlong Zheng ${ }^{\dagger}$, Duojie $\mathrm{Wu}^{\$}$, Shuomeng Zhang ${ }^{\dagger}$, Meng $\mathrm{Gu}^{\$}$, Qinggang $\mathrm{He}$ $\dagger, \%, *$

${ }^{\dagger}$ College of Chemical and Biological Engineering, Zhejiang Provincial Key Laboratory of Advanced Chemical Engineering Manufacture Technology, Zhejiang University, Hangzhou, Zhejiang 310027, China

\$ Department of Materials Science and Engineering, Guangdong Provincial Key Laboratory of Energy Materials for Electric Power, Southern University of Science and Technology, Shenzhen, 518055, China

${ }^{\%}$ Ningbo Research Institute, Zhejiang University, Ningbo, Zhejiang 315100, China

*E-mail: qghe@zju.edu.cn (Q.H.)

Materials 
The graphene oxide (GO) dispersion (10 mg/g) was provided by Gaoxi Tech Co. Ltd. The nitrogenous compounds $\left(\mathrm{C}_{3} \mathrm{~N}_{3}\left(\mathrm{NH}_{2}\right)_{3}\right)$ and inorganic iron salts $\left(\mathrm{FeCl}_{2} \cdot 4 \mathrm{H}_{2} \mathrm{O}\right)$ were purchased from Sinopharm Chemical Reagent Co. Ltd. Concentrated HCl (36 w.\%) were also from Sinopharm Chemical Reagent Co. Ltd. High purity oxygen, argon and ammonia (5v.\% in Ar), argon and hydrogen ( 5 v. \% in Ar) were supported by Nanjing Special Gas Factory Co. Ltd. The deionized water was prepared by a SMART ultra-pure water system.

\section{Preparation of rGO}

Reduced graphene oxide was obtained by thermal reduction of graphene oxide. ${ }^{1}$ GO were thermally reduced in a horizontal tube furnace under argon and hydrogen (5 v. $\%$ in Ar) at $900{ }^{\circ} \mathrm{C}$ for $2 \mathrm{~h}$. The heating rate was $5{ }^{\circ} \mathrm{C} / \mathrm{min}$.

\section{Preparation of GO-N and rGO-N}

GO-N and rGO-N catalysts were prepared following previous studies. ${ }^{2} \mathrm{GO}$ dispersion $(10 \mathrm{~g})$ was dissolved in $100 \mathrm{~mL}$ of ultra-pure water. After ultrasonic treatment for 15 minutes, the mixture was subjected to continuous electromagnetic stirring to form a homogeneous GO dispersion. $80 \mathrm{mg}$ of melamine was dissolved in 8 $\mathrm{mL}$ of ultra-pure water at $80{ }^{\circ} \mathrm{C}$ and added drop by drop into the $\mathrm{GO}$ solution. The mixture was freeze-dried for 72 hours to form a 3D porous structure. Finally, the obtained 3D graphene-based catalysts were pyrolyzed in a tube furnace under argon and ammonia $\left(5 \mathrm{v} . \%\right.$ in Ar) at the required temperature $\left(750{ }^{\circ} \mathrm{C}, 5^{\circ} \mathrm{C} / \mathrm{min}\right)$ for $3 \mathrm{~h}$. The resulting sample is expressed as GO-N. The $100 \mathrm{mg}$ prepared $\mathrm{rGO}$ was dissolved in $100 \mathrm{~mL}$ of ultra-pure water to prepare $\mathrm{rGO}-\mathrm{N}$ in the same way.

\section{Preparation of GO-Fe-N and rGO-Fe-N}

Ultra-pure water containing $\mathrm{FeCl}_{2} \cdot 4 \mathrm{H}_{2} \mathrm{O}(54 \mathrm{mg})$ was added into the GO-N and rGO-N mixture solution under vigorous stirring. The resulting mixture was then stirred for another 1 hour. After sufficient reaction, the mixture was freeze-dried for 72 hours to form a 3D porous structure. Subsequently, the obtained black powder was pyrolyzed at $750{ }^{\circ} \mathrm{C}$ for $3 \mathrm{~h}$ with a heating rate of $5{ }^{\circ} \mathrm{C} \mathrm{min}^{-1}$ under the argon and ammonia $(5 \mathrm{v} . \%$ in Ar) flow in order to form the Fe-N-C active sites on the supports. Finally, the obtained solid was washed with $0.1 \mathrm{M} \mathrm{HCl}$ for $12 \mathrm{~h}$, followed by drying at $60{ }^{\circ} \mathrm{C}$ in a vacuum 
oven overnight. The final GO-Fe-N and rGO-Fe-N catalysts were obtained under the second round of pyrolysis. The condition was consistent with the first pyrolysis. ${ }^{3-5}$

\section{KPFM measurement}

Both sides of the $\mathrm{SiO}_{2} / \mathrm{Si}$ substrate are covered with $\mathrm{Au}$ films for grounding. The graphene-based catalysts must be uniformly dispersed and transferred to the $\mathrm{SiO}_{2} / \mathrm{Si}$ substrate to obtain accurate CPD measurements for all graphene-based samples that still have individual lamellar nanostructures. A drop-casting method was used in a twostep process. Graphene-based catalysts $(5 \mathrm{mg})$ were mixed with a solution of ethanol $(10 \mathrm{~mL})$ in water $(20 \mathrm{~mL})$ followed by $45 \mathrm{~min}$ of sonication for dispersion. Then $1 \mathrm{~mL}$ of the above solution was transferred into a vial and $5 \mathrm{ml}$ of ethanol was added. After

sonication for $1 \mathrm{~h}, 5 \mu \mathrm{L}$ of the solution were transferred onto a $2 \times 2 \mathrm{~cm}^{2}$ substrate using a pipette. After the solvent evaporated, AFM topography tests were first performed to confirm the distribution and clarify the ideal location of the KPFM. 


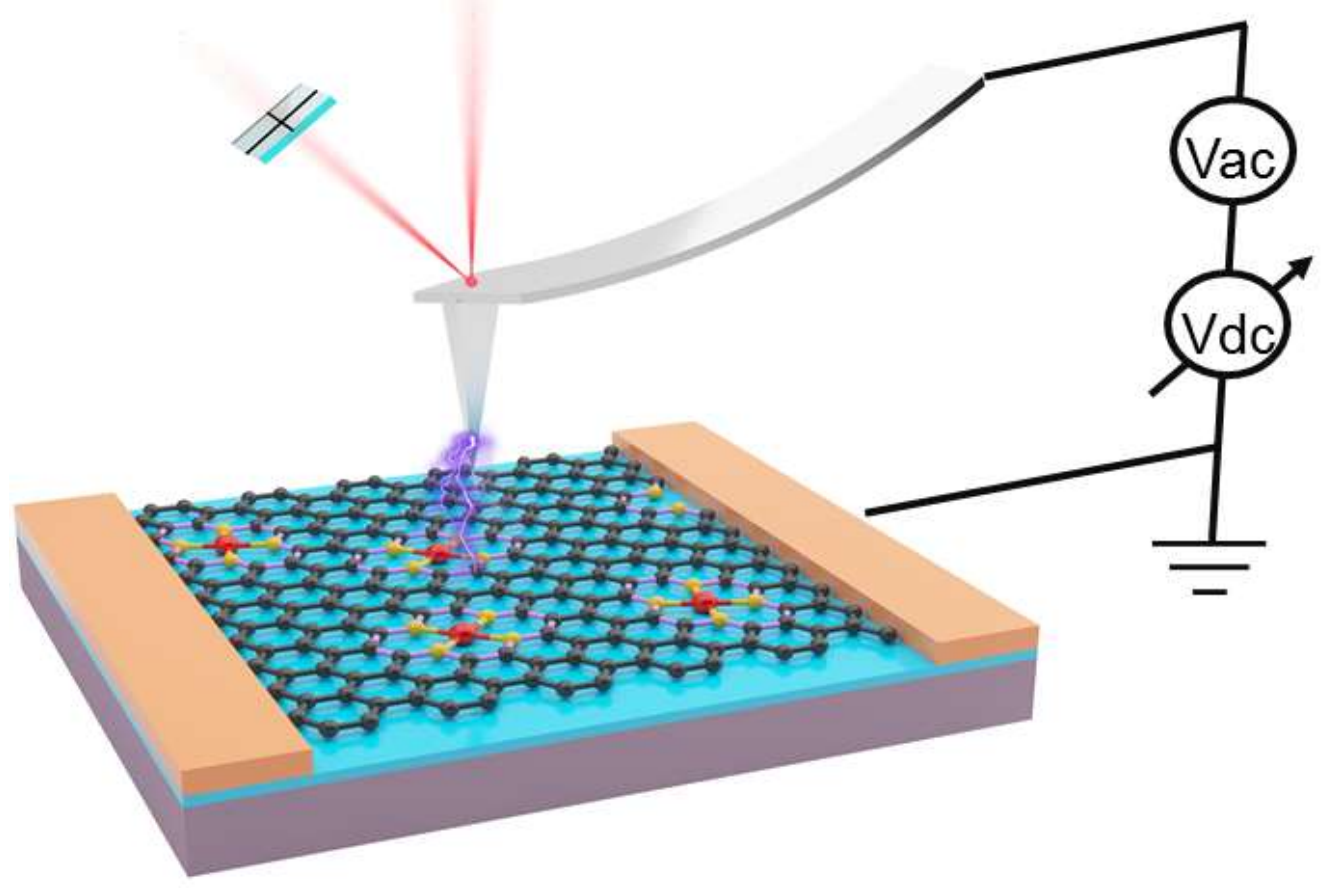

Figure S1. A schematic diagram of KPFM test device. 


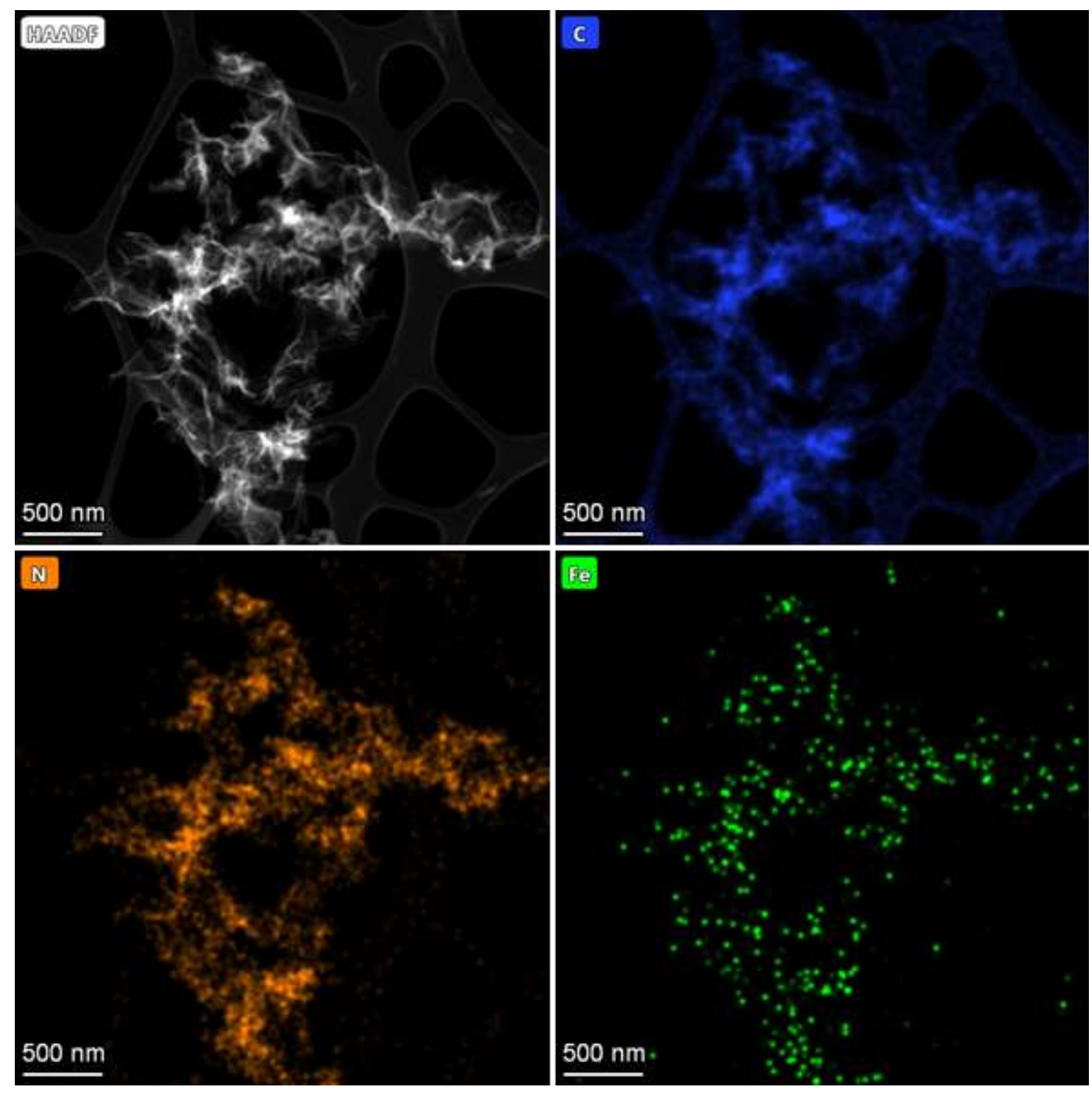

Figure S2. HAADF-STEM images of GO-Fe-N and corresponding elemental mapping images exhibiting the distribution of $\mathrm{C}$ (blue), $\mathrm{N}$ (orange), and Fe (green). 


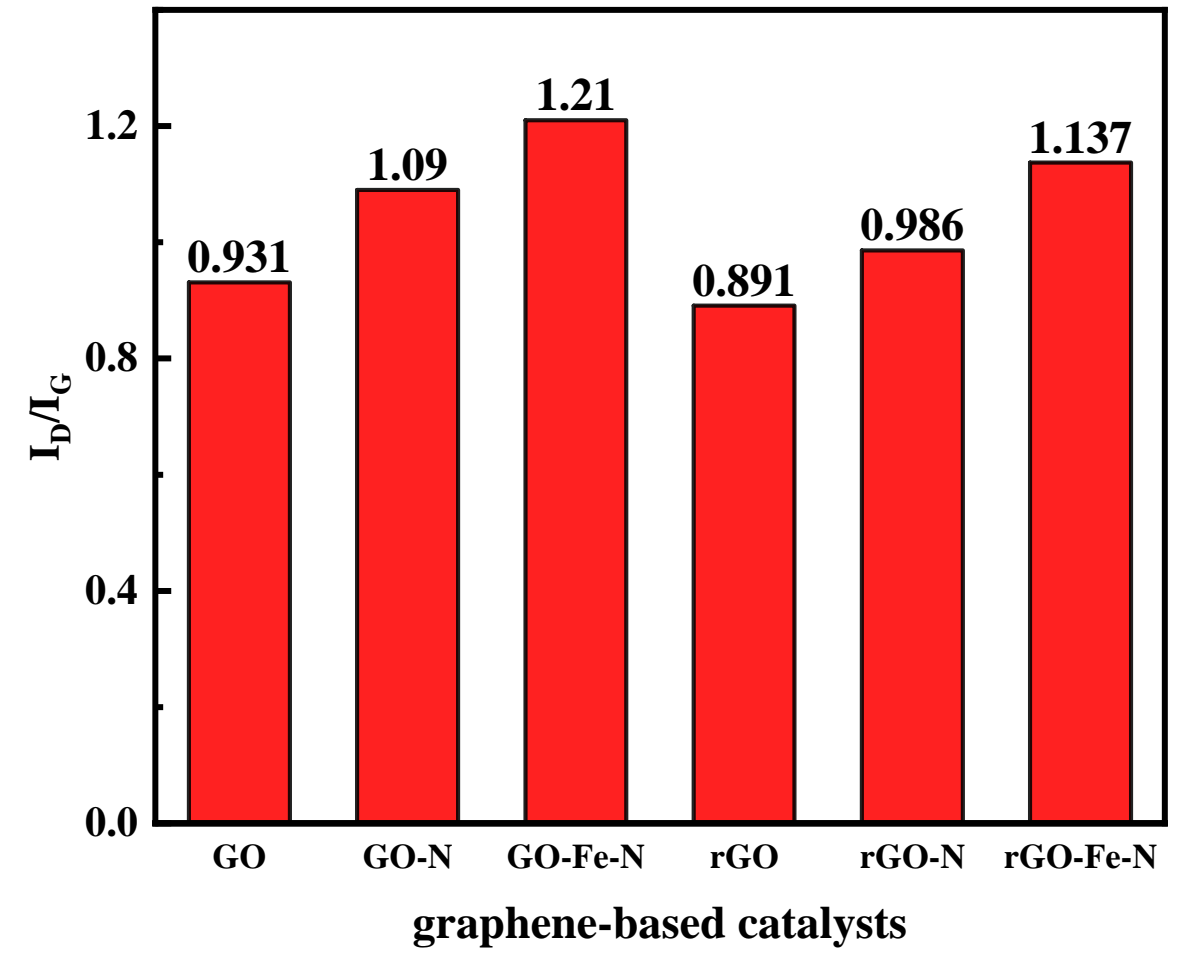

Figure S3. $\mathrm{I}_{\mathrm{D}} / \mathrm{I}_{\mathrm{G}}$ values of graphene-based catalysts in Raman spectra 


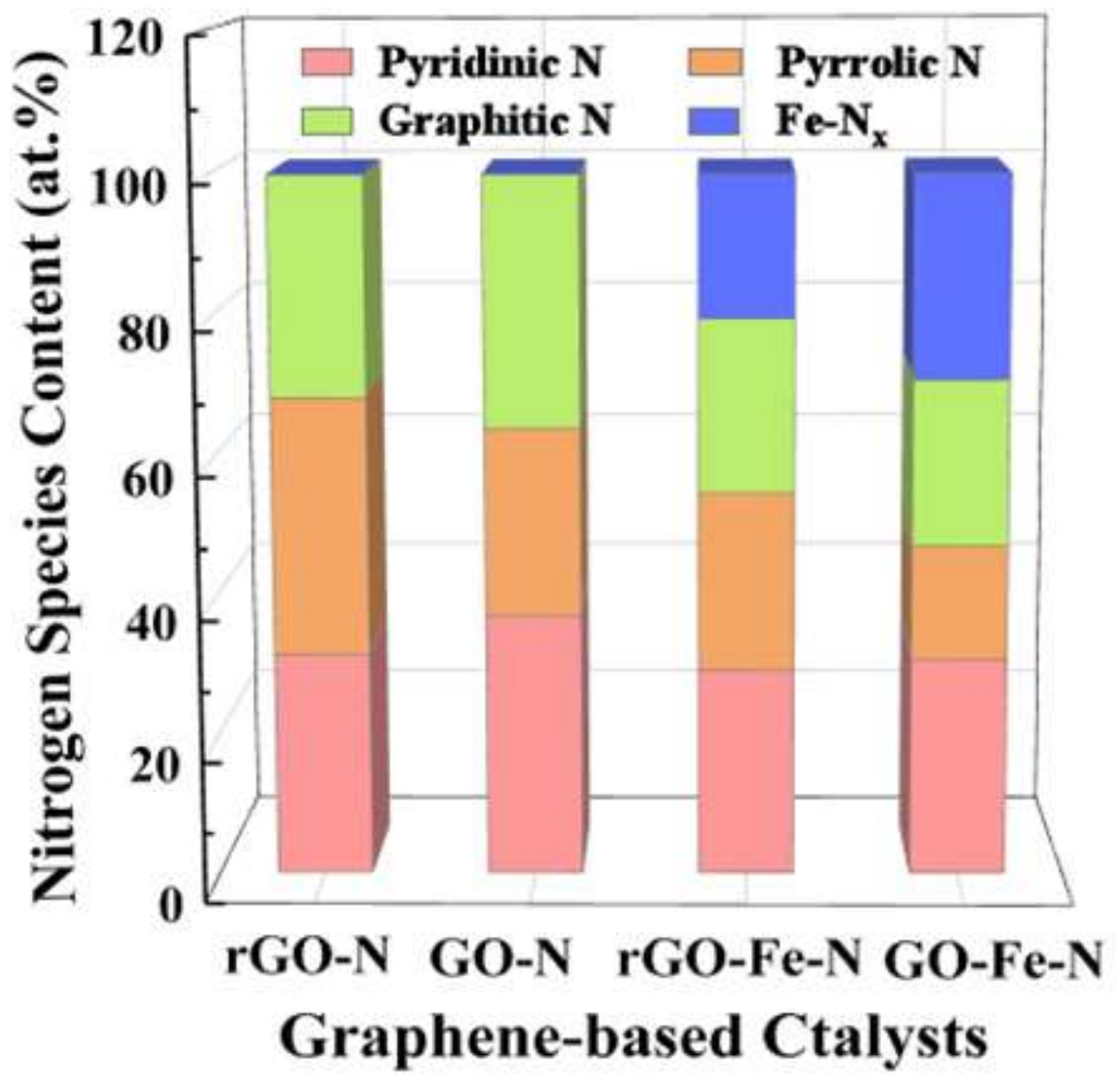

Figure S4. The atomic percentage of pyridinic-N, pyrrolic-N, graphitic-N, and Fe-N of graphene-based catalysts. 

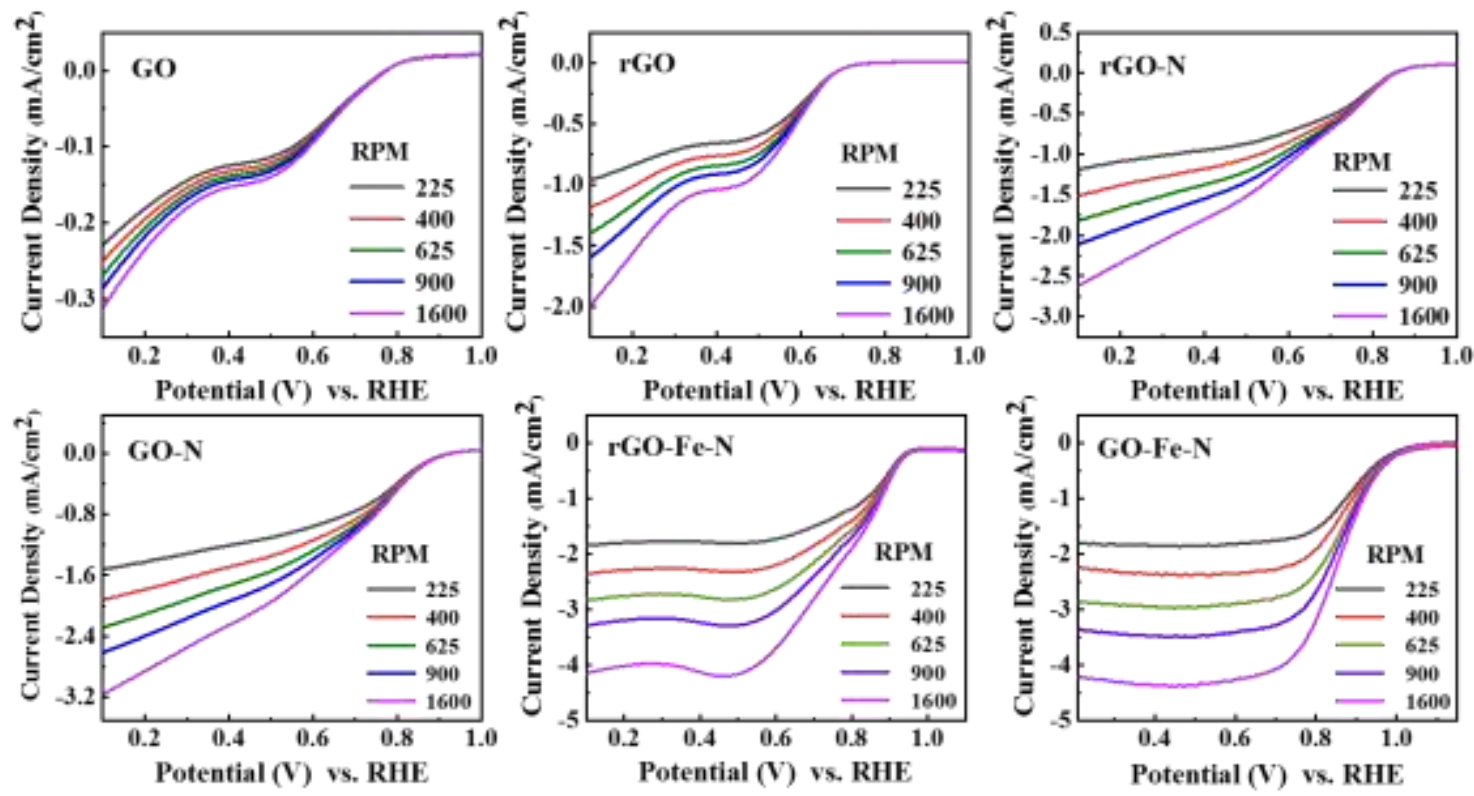

Figure S5. ORR polarization curves of graphene-based catalysts at different rotating speeds. 

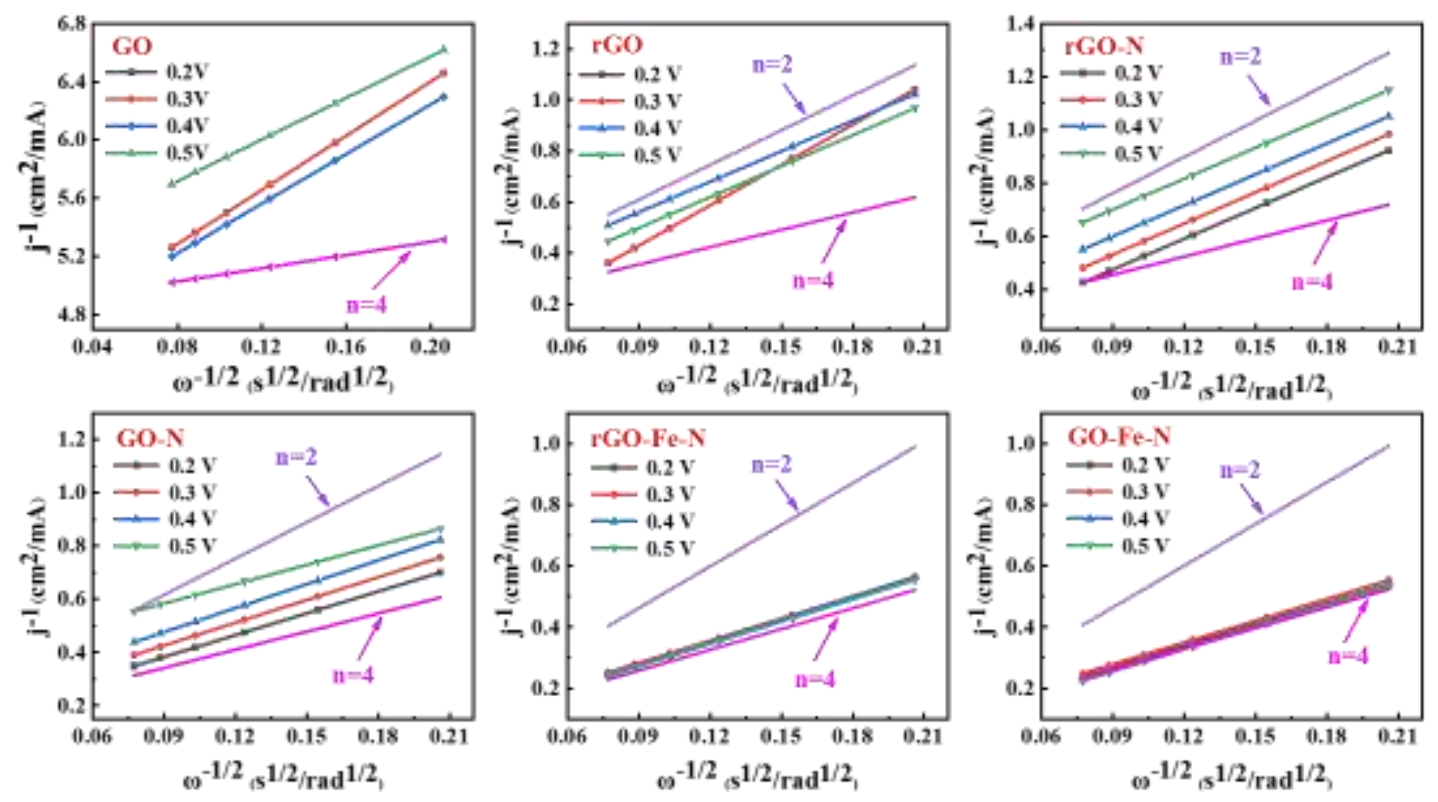

Figure S6. K-L plots of the current density reciprocal $\left(j^{-1}\right)$ vs. $\omega^{-1 / 2}$ at different potentials in graphene-based catalysts. 


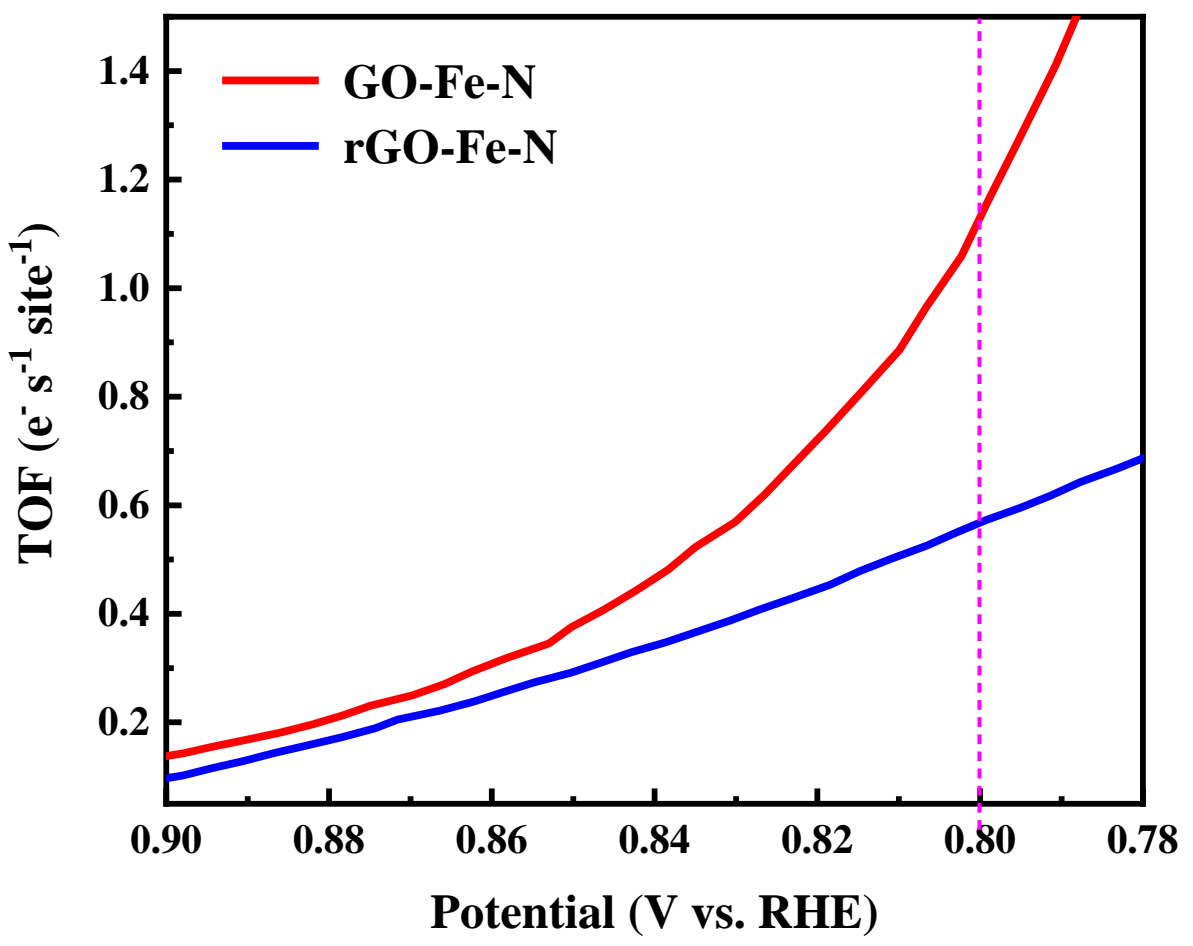

Figure S7. TOF of the Fe site in GO-Fe-N and rGO-Fe-N. 

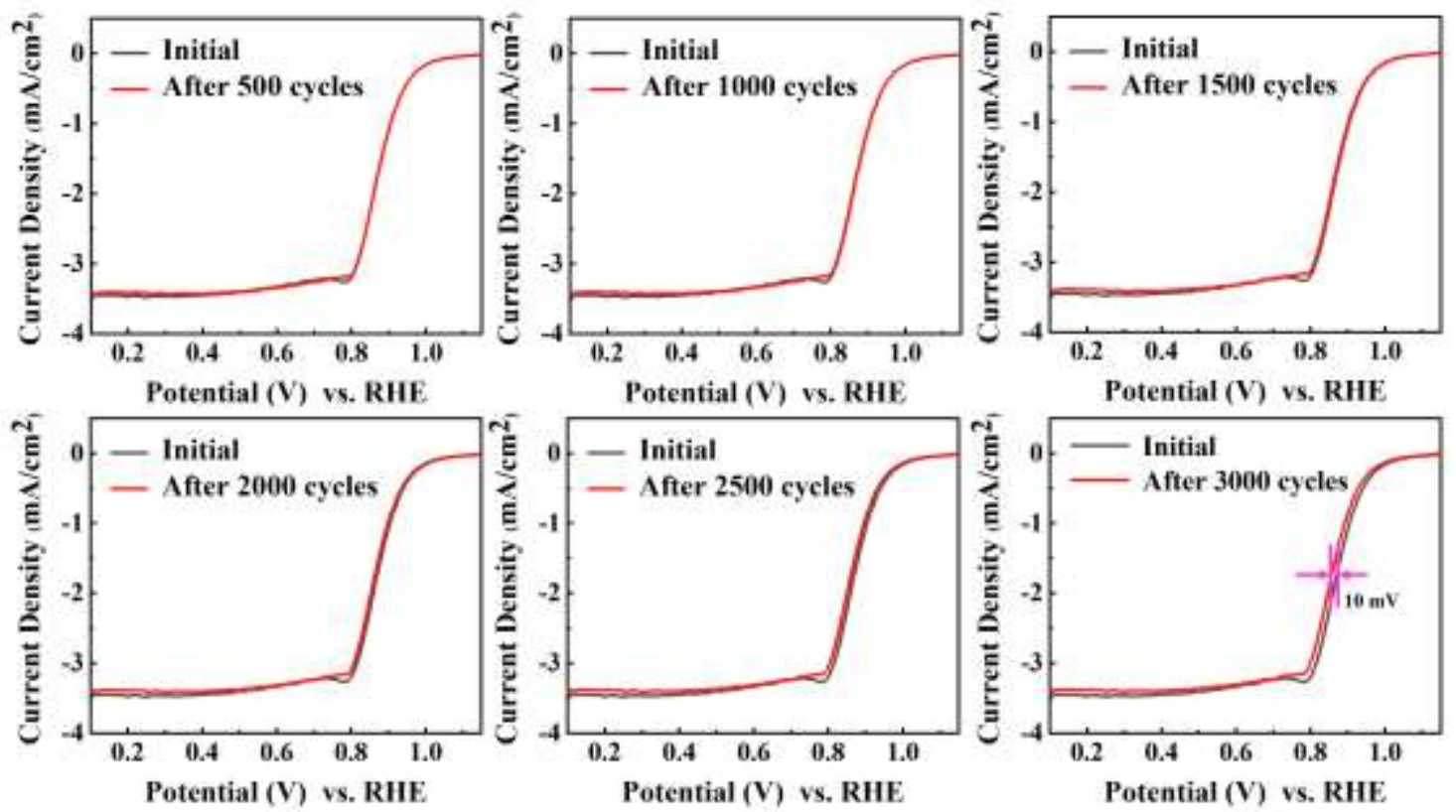

Figure S8. LSV curves of GO-Fe-N catalyst before and after different potential cycles at $900 \mathrm{rpm}$. Potential cycling between 0.4 to $1.0 \mathrm{~V}$ vs RHE in $0.1 \mathrm{M} \mathrm{KOH} \mathrm{O}_{2}$-saturated solution. 

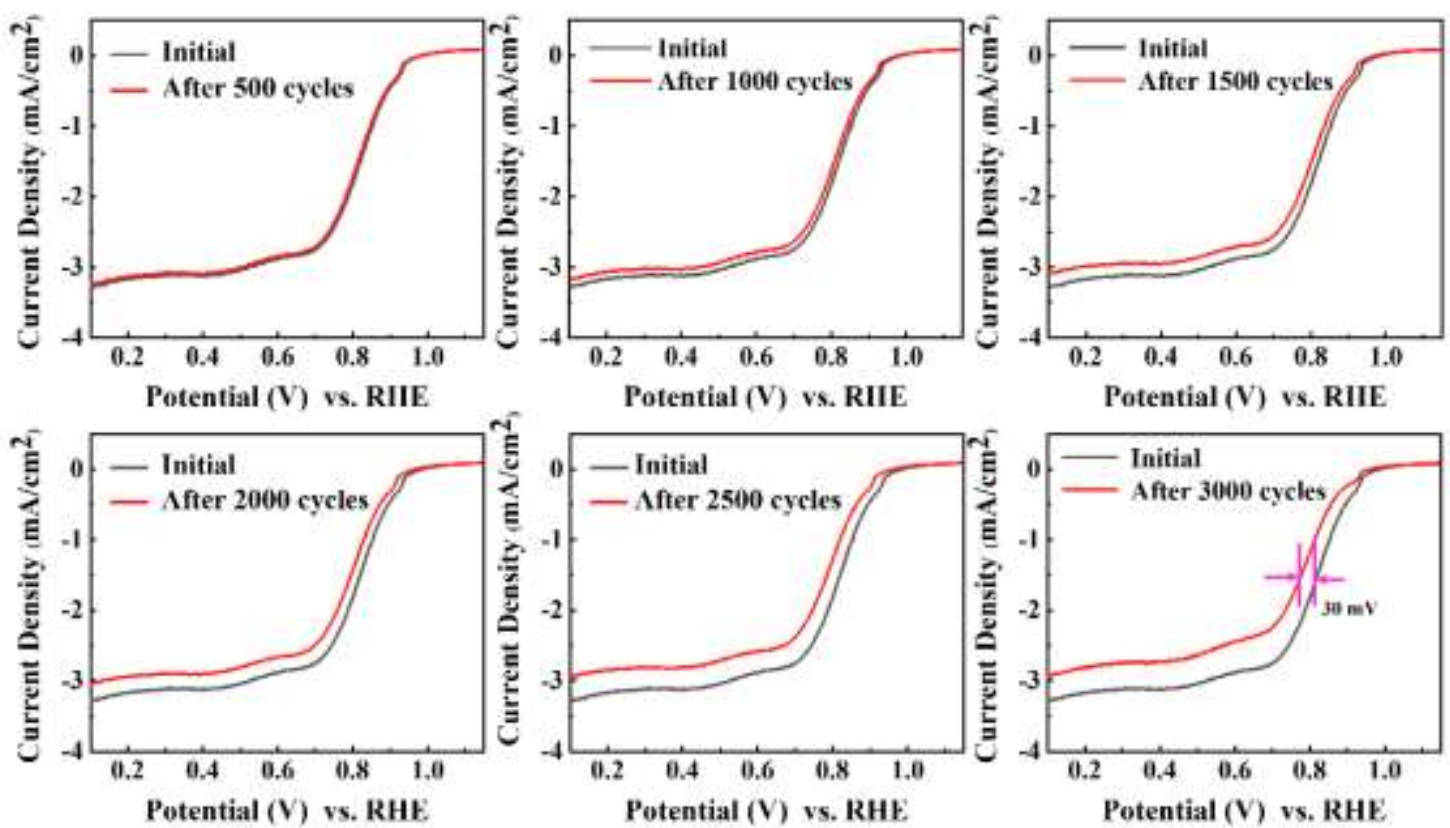

Figure S9. LSV curves of rGO-Fe-N catalyst before and after different potential cycles at $900 \mathrm{rpm}$. Potential cycling between 0.4 to $1.0 \mathrm{~V}$ vs $\mathrm{RHE}$ in $\mathrm{O}_{2}$-saturated $0.1 \mathrm{M} \mathrm{KOH}$ solution. 

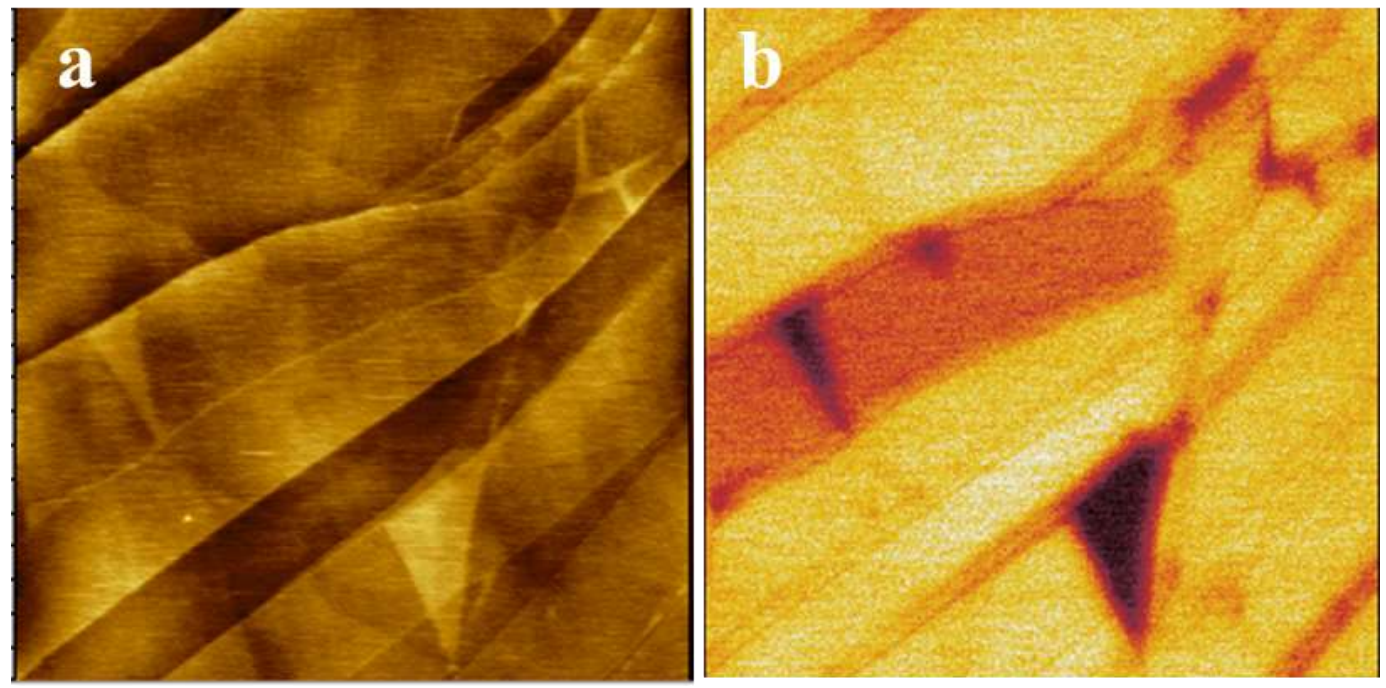

Figure S10. (a) Topography of HOPG deposited on a $\mathrm{SiO}_{2} / \mathrm{Si}$ substrate, (b) the contact potential map obtained using KPFM. 


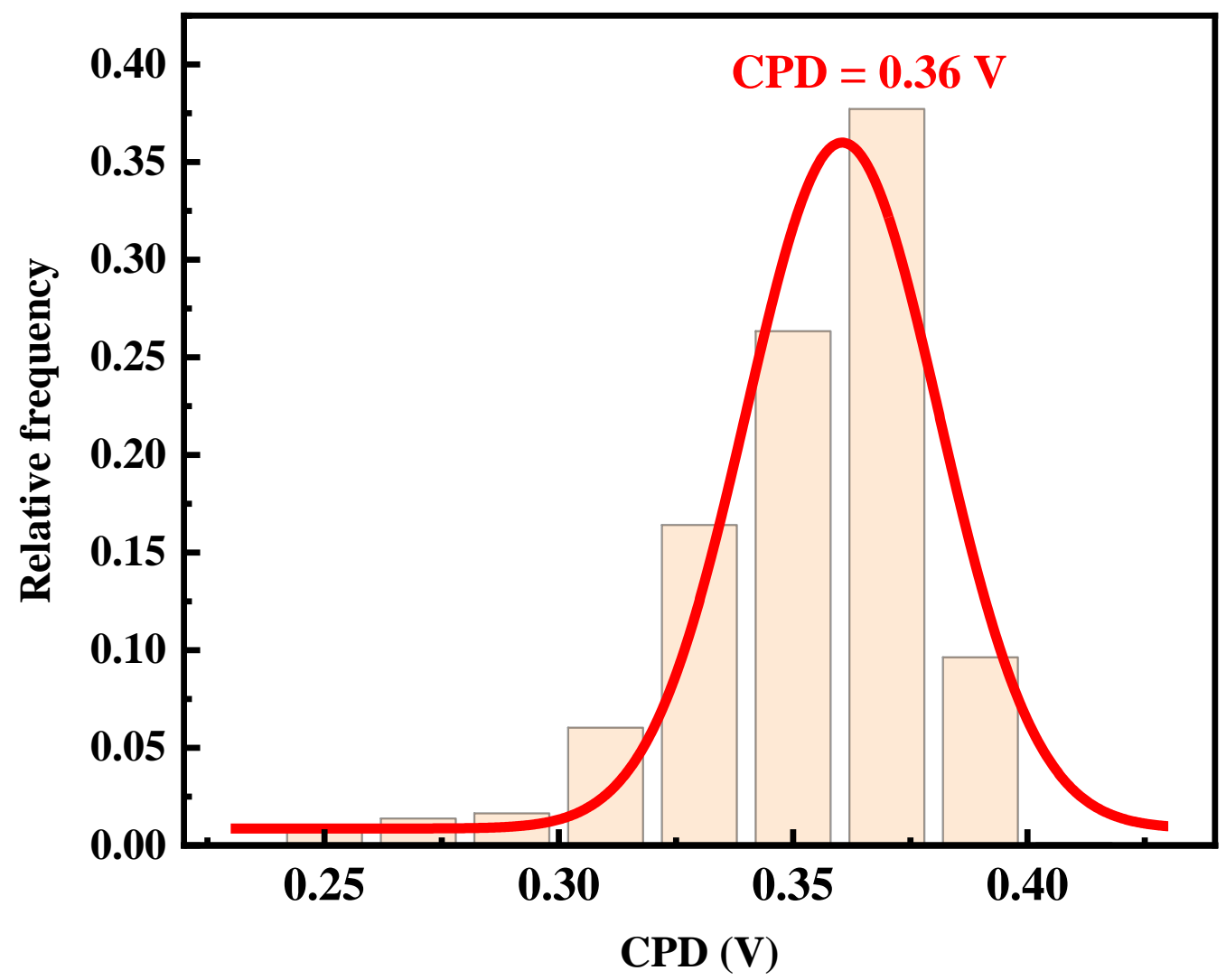

Figure S11. Distribution of the CPD of Kelvin signals in the HOPG. 

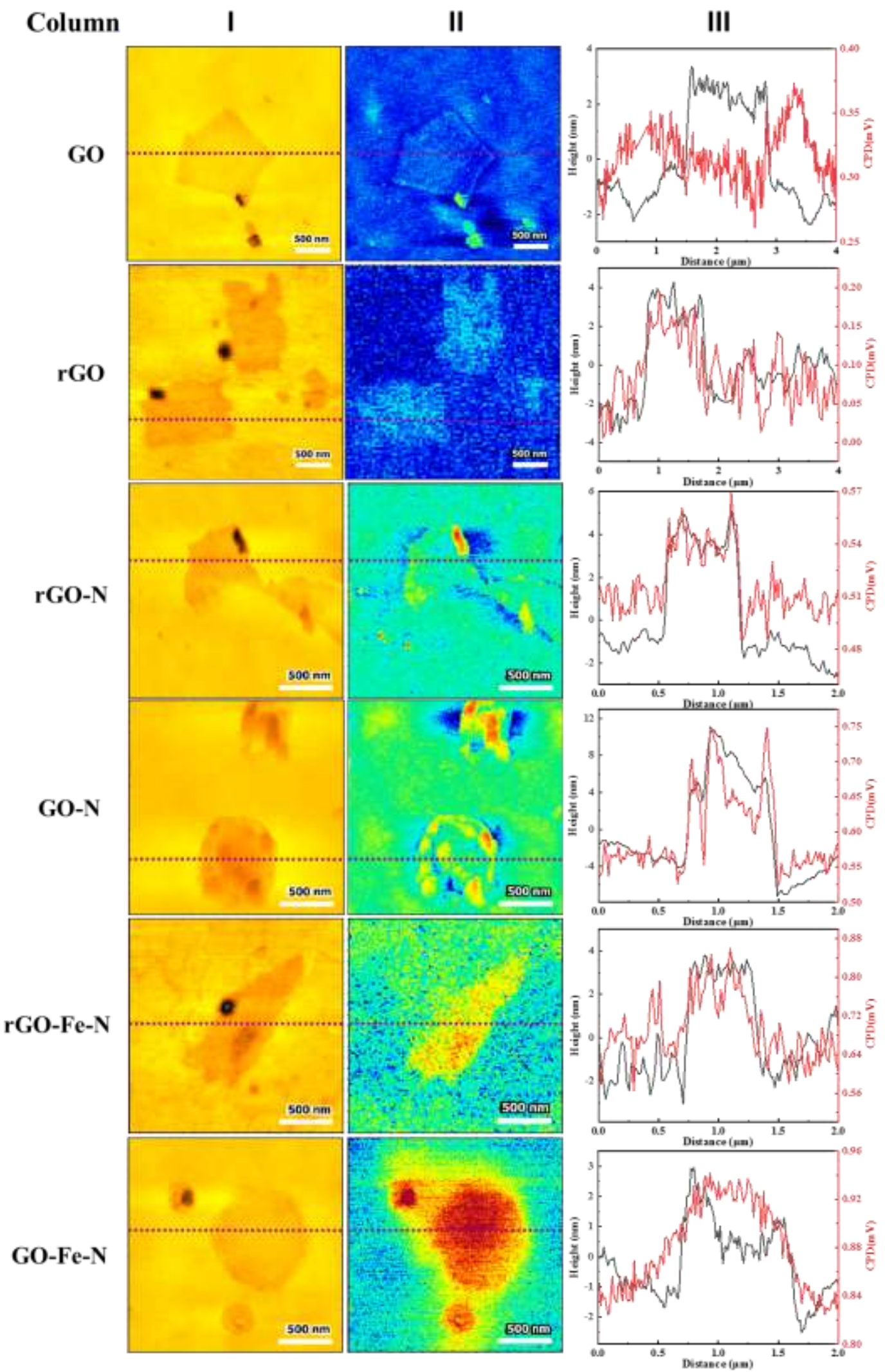

Figure S12. Topography of graphene-based catalysts deposited on the $\mathrm{SiO}_{2} / \mathrm{Si}$ substrate (Column I), the CPD images of graphene-based catalysts (Column II) and the height and CPD curves following the dashed lines (Column III). 


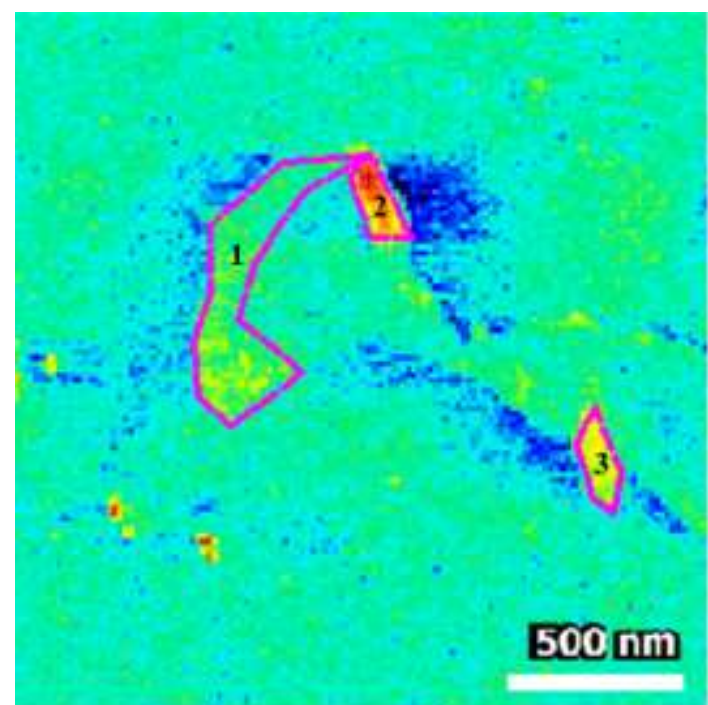

rGO-N

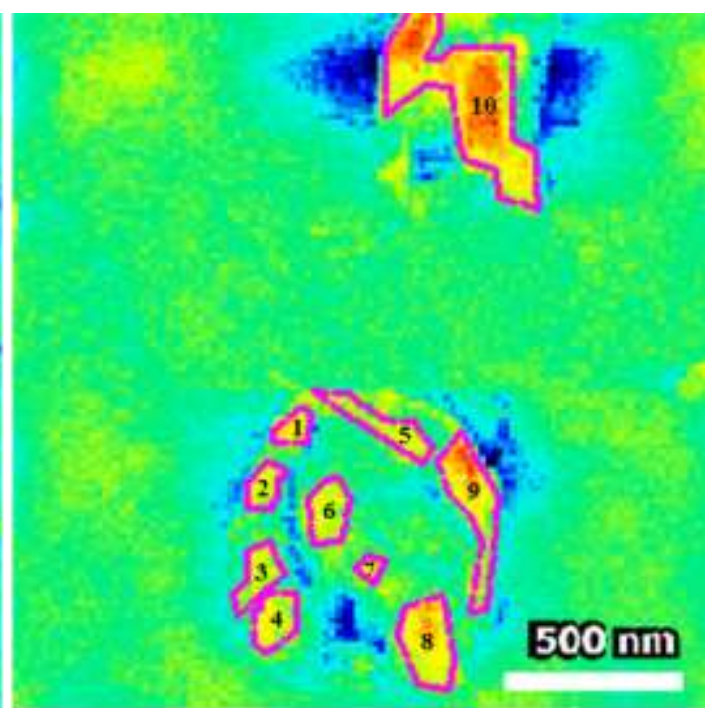

GO-N

Figure S13. The regions with CPD values higher than the average for GO-N and rGO$\mathrm{N}$ (calculated using AutoCAD). The average CPD values on the surface of the rGO-N and GO-N samples are $0.56 \mathrm{mV}$ and $0.67 \mathrm{mV}$, respectively. 
Table S1. The chemical composition of graphene-based catalysts from SEM-EDS

\begin{tabular}{ccccc}
\hline Sample & C Wt. \% & O Wt. \% & N Wt. \% & Fe Wt. \% \\
\hline GO & 55.79 & 44.21 & 0 & 0 \\
rGO & 96.20 & 3.80 & 0 & 0 \\
GO-N & 88.09 & 6.72 & 5.19 & 0 \\
rGO-N & 93.55 & 3.21 & 3.24 & 0 \\
GO-Fe-N & 87.37 & 4.58 & 5.83 & 2.22 \\
rGO-Fe-N & 91.22 & 3.08 & 4.18 & 1.52 \\
\hline
\end{tabular}

Table S2. Atomic percentages of different N species in the graphene-based catalysts from N 1s XPS spectra.

\begin{tabular}{ccccc}
\hline Sample & pyridinic N & graphitic N & pyrrolic N & Fe- $\mathrm{N}_{\mathrm{x}}$ \\
\hline GO-N & $37.47 \%$ & $35.73 \%$ & $26.80 \%$ & 0 \\
rGO-N & $31.74 \%$ & $31.37 \%$ & $36.89 \%$ & 0 \\
GO-Fe-N & $31.15 \%$ & $23.67 \%$ & $16.18 \%$ & $29.00 \%$ \\
rGO-Fe-N & $29.64 \%$ & $24.68 \%$ & $25.45 \%$ & $20.23 \%$ \\
\hline
\end{tabular}


Table S3. Binding energies and atomic percentages of different Fe species in rGO-Fe$\mathrm{N}$ and GO-Fe-N from Fe 2p XPS spectra.

\begin{tabular}{|c|c|c|c|c|c|c|c|c|}
\hline \multirow{3}{*}{$\begin{array}{c}\text { Classification } \\
\text { Binding }\end{array}$} & \multicolumn{4}{|c|}{ rGO-Fe-N } & \multicolumn{4}{|c|}{ GO-Fe-N } \\
\hline & \multicolumn{2}{|c|}{$\mathrm{Fe}^{2+}$} & \multicolumn{2}{|c|}{$\mathrm{Fe}^{3+}$} & \multicolumn{2}{|c|}{$\mathrm{Fe}^{2+}$} & \multicolumn{2}{|c|}{$\mathrm{Fe}^{3+}$} \\
\hline & $2 \mathrm{p}_{3 / 2}$ & $2 \mathrm{p}_{1 / 2}$ & $2 p_{3 / 2}$ & $2 p_{1 / 2}$ & $2 p_{3 / 2}$ & $2 \mathrm{p}_{1 / 2}$ & $2 p_{3 / 2}$ & $2 p_{1 / 2}$ \\
\hline energy / eV & 710.0 & 723.2 & 714.6 & 725.4 & 710.0 & 723.2 & 714.6 & 725.4 \\
\hline Proportion / \% & \multicolumn{2}{|c|}{75.40} & \multicolumn{2}{|c|}{24.60} & \multicolumn{2}{|c|}{63.10} & \multicolumn{2}{|c|}{36.90} \\
\hline
\end{tabular}

Table S4. EXAFS fitting parameters at the Fe K-edge for rGO-Fe-N and GO-Fe-N $\left(\mathrm{S}_{0}^{2}=0.78\right)$.

\begin{tabular}{ccccccc}
\hline Sample & Path & $\mathrm{CN}$ & $\mathrm{R} / \AA$ & $\sigma^{2} / \AA^{2}$ & $\Delta \mathrm{E}_{0} / \mathrm{eV}$ & $\mathrm{R}$-factor \\
\hline rGO-Fe-N & Fe-N & $3.2 \pm 0.2$ & $1.96 \pm 0.01$ & $0.007 \pm 0.001$ & $-0.7 \pm 0.6$ & 0.002 \\
GO-Fe-N & Fe-N & $4.2 \pm 0.3$ & $1.95 \pm 0.02$ & $0.007 \pm 0.003$ & $-2.9 \pm 2.1$ & 0.008 \\
\hline
\end{tabular}

Note: $\mathrm{CN}$ : coordination number, R: distance between the absorber and backscatter atoms, $\sigma^{2}$ :

Debye-Waller factors, $\Delta \mathrm{E}_{0}$ : the inner potential correction, $\mathrm{R}$-factor: goodness of fit.

Table S5. Comparison of electrochemical performance parameters of graphene-based catalysts for ORR.

\begin{tabular}{ccccc}
\hline Catalyst & $\mathrm{E}_{\text {onset }} / \mathrm{V}$ & $\mathrm{E}_{1 / 2} / \mathrm{V}$ & $\mathrm{n}$ & Tafel slope/ $\mathrm{mV} \mathrm{dec}^{-1}$ \\
\hline rGO-N & 0.85 & 0.62 & 3.07 & $70.3 / 221.8$ \\
GO-N & 0.94 & 0.64 & 3.76 & $67.3 / 204.6$ \\
rGO-Fe-N & 0.96 & 0.81 & 3.82 & $66.2 / 132.7$ \\
GO-Fe-N & 1.05 & 0.87 & 3.93 & $66.2 / 117.4$ \\
\hline
\end{tabular}


Table S6. The high CPD area in GO-N and rGO-N from Figure S11

\begin{tabular}{|c|c|c|}
\hline Sample & $\mathrm{rGO}-\mathrm{N} / \mu \mathrm{m}^{2}$ & $\mathrm{GO}-\mathrm{N} / \mu \mathrm{m}^{2}$ \\
\hline Area 1 & 0.125 & 0.008 \\
\hline Area 2 & 0.021 & 0.010 \\
\hline Area 3 & 0.020 & 0.014 \\
\hline Area 4 & / & 0.016 \\
\hline Area 5 & l & 0.019 \\
\hline Area 6 & / & 0.016 \\
\hline Area 7 & l & 0.004 \\
\hline Area 8 & l & 0.031 \\
\hline Area 9 & l & 0.035 \\
\hline Area 10 & l & 0.103 \\
\hline $\begin{array}{c}\text { Total Area with } \\
\text { high CPD }\end{array}$ & 0.166 & 0.256 \\
\hline $\begin{array}{c}\text { Total Area of } \\
\text { sample }\end{array}$ & 0.611 & 0.669 \\
\hline
\end{tabular}




\section{Reference:}

(1) Zhao, B.; Liu, P.; Jiang, Y.; Pan, D.; Tao, H.; Song, J.; Fang, T.; Xu, W. Supercapacitor Performances of Thermally Reduced Graphene Oxide. Journal of Power Sources. 2012, 198, 423-427.

(2) Li, X.; Wang, H.; Robinson, J. T.; Sanchez, H.; Diankov, G.; Dai, H. Simultaneous Nitrogen Doping and Reduction of Graphene Oxide. Journal of the American Chemical Society. 2009, 131, 15939-15944.

(3) Wang, X.; Jia, Y.; Mao, X.; Liu, D.; He, W.; Li, J.; Liu, J.; Yan, X.; Chen, J.; Song, L.; Du, A.; Yao, X. Edge-Rich Fe-N4 Active Sites in Defective Carbon for Oxygen Reduction Catalysis. Advanced Materials. 2020, 32, 2000966.

(4) Hu, Y.; Yang, R.; Chen, H.; Han, S.; Wang, J.; Wang, X.; Deng, X.; He, C.-H.; He, Q.; Jiang, Z.; Gu, M. One-Pot Synthesis of a Highly Active 3-Dimensional $\mathrm{Fe}-\mathrm{Nx}-\mathrm{Cnts} /$ Rgo Composite Catalyst for Oxygen Reduction. ChemElectroChem. 2019, 6, 504-513.

(5) Deng, X.; Xiao, M.; Yang, R.; Guo, F.; Chen, H.; Hu, Y.; Li, Y.; Zhu, C.; Deng, Y.; Jiang, Z.; Xu, Z.; Gao, C.; He, Q.; Ge, J.; Hou, Y.; Zhang, X.; Chen, Z. The Effect of Cnts on Performance Improvement of rGO Supported Fe-Nx/C Electrocatalysts for the Oxygen Reduction Reaction. Journal of The Electrochemical Society. 2018, 165, F401F407. 